\title{
Problems in detecting mosaic DNA methylation in Angelman syndrome
}

\author{
European Journal of Human Genetics (2003) 11, 913-915. doi:10.1038/sj.ejhg.5201078 \\ Published online 1 October 2003
}

The Prader-Willi and Angelman syndromes (PWS and AS) are neurogenetic disorders that are caused by the loss of function of imprinted genes on the proximal long arm of chromosome 15. ${ }^{1}$ Deletions, uniparental disomy and imprinting defects, which account for most cases, can easily be detected by determining the DNA methylation pattern in proximal 15q. The best studied site is the promotor region of the SNRPN gene, which is methylated on the maternal chromosome and unmethylated on the paternal chromosome. ${ }^{2}$ Initially, Southern blot analysis of DNA digested with methylation-sensitive restriction enzymes was used to study DNA methylation. In recent years, most laboratories have switched over to methylationspecific PCR (MS-PCR). ${ }^{3}$ MS-PCR is based on the conversion of cytosine, but not 5-methyl-cytosine, to uracil by treatment with sodium bisulfite. By this, allelic methylation differences are converted into sequence differences, which can be detected by specific PCR reactions. Two different primer combinations are currently in use in most laboratories: Kubota et l $^{4}$ developed two specific primer pairs, one for the methylated maternal allele and the other for the unmethylated paternal allele. The reactions can be performed separately (simplex PCR) or in combination (duplex PCR). Zeschnigk et $a l^{5}$ designed one common primer that anneals to both alleles and one specific primer each for the methylated and the unmethylated allele. The three primers are always used together in one reaction.

Within the German external quality assessment scheme for the molecular diagnosis of PWS and AS, once a yearcoded DNA samples $(5-10 \mu \mathrm{g})$ from three core families are sent to laboratories in Germany, Austria and Switzerland. In 2001 and 2002, 17 and 22 labs, respectively, participated. The labs were asked to check the clinical diagnosis by a methylation test of their choice. Approximately 50\% of the labs used the Kubota test and 50\% used the Zeschnigk test. The original data and reports were evaluated by $\mathrm{BH}, \mathrm{CL}, \mathrm{KB}, \mathrm{JB}$ and $\mathrm{BJ}$.

In both years, one child was normal, one child had PWS and one child had AS. The 2001 PWS patient had uniparental disomy and the 2002 PWS patient had a deletion. Apart from one lab, which obtained a falsenegative result in the 2001 PWS patient, all labs found both alleles in the normal child and a methylated allele only in the patients with PWS (data not shown).
The two patients with AS had a sporadic imprinting defect without an IC deletion. As found by Southern blot analysis (Figure 1), the patients have a faint methylated band. This indicates that they are mosaic for an imprinting defect that occurred after fertilization. ${ }^{6}$ In 2001, four labs, and in 2002, seven labs reported a false-negative result ('normal methylation pattern') in the patient with AS. The seven labs in 2002 include two of the four labs that failed in 2001. As shown in Table 1, each of the labs reporting a false result had used the methylation test described by Kubota et al. Most of these labs had performed simplex reactions. Although some labs had also performed duplex reactions and obtained bands of unequal intensity, they were apparently misled by the presence of a strong PCR product in the simplex reactions. In contrast, all labs reporting the correct result had used the methylation test described by Zeschnigk et al (six labs in 2001 and 10 labs in 2002) or another technique (two labs in 2001 and one lab in 2002). Depending on the PCR efficiency in the different labs, these labs had obtained no methylated band, or a faint methylated band (data not shown). Typical MS-PCR results are shown in Figure 2.

The fact that 4/8 (50\%) and 7/11 (64\%), respectively, of the labs who used the Kubota test in 2001 and 2002 obtained false-negative results in the AS patients demonstrates that this test, especially when simplex reactions are performed, is of insufficient sensitivity in the case of mosaic DNA methylation. These patients have a small fraction of normal cells with a methylated maternal allele. It is likely that amplification products from this allele, although under-represented in genomic DNA, are driven onto a plateau, because a specific primer pair for methylated DNA is used. The problem can be diminished by performing a duplex reaction. We note in this context that a commercially available kit for the Kubota test includes a protocol for simplex reactions only, although the original publication $^{4}$ describes simplex and duplex reactions. It should be noted that simplex reactions for the methylated and unmethylated alleles have another disadvantage in that a PCR failure in one tube will automatically lead to a false-positive result.

In contrast to the Kubota test, the test described by Zeschnigk et al is based on a duplex PCR with one common primer, for which the two reactions compete. Thus, the test 
Table 1 Number of labs reporting correct and incorrect results in AS patients

\begin{tabular}{|c|c|c|c|c|c|c|}
\hline Result & Kubota simplex & Kubota duplex & Kubota simplex+duplex & Zeschnigk & Zeschnigk+Kubota duplex & Others \\
\hline \multicolumn{7}{|l|}{2001} \\
\hline AS (correct) & - & 3 & 1 & 6 & 1 & $2^{a}$ \\
\hline Normal (incorrect) & 2 & - & 2 & - & - & - \\
\hline \multicolumn{7}{|l|}{2002} \\
\hline AS (correct) & - & 3 & 1 & 10 & - & $1^{\mathrm{b}}$ \\
\hline Normal (incorrect) & 5 & 1 & 1 & - & - & - \\
\hline
\end{tabular}

ane lab performed MS-PCR/DHPLC analysis of bisulfite-treated DNA. ${ }^{7}$ The other lab performed two independent tests: (i) PCR amplification of DNA digested with $M c r B C$ and Notl, respectively, ${ }^{8}$ and (ii) duplex MS-PCR according to Kubota et al. This lab is listed only in this column. ${ }^{b} \mathrm{MS}-\mathrm{PCR} / \mathrm{DHPLC}$ analysis of bisulfite-treated DNA. ${ }^{7}$

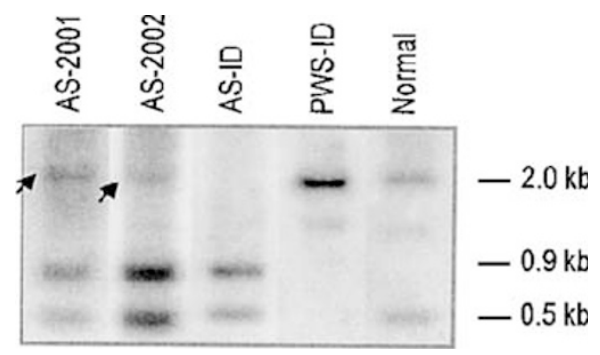

Figure 1 Methylation-sensitive Southern blot analysis. Genomic DNA samples from the two AS patients (AS2001 and AS-2002), another AS patient with an imprinting defect (AS-ID), a PWS patient with an imprinting defect (PWS-ID) as well as a normal control were digested with Bglll + Hpall, and analyzed by Southern blot hybridization with a probe for SNRPN, as described by Buiting et al. ${ }^{10}$ Note that the AS-2001 and AS-2002 patients have a faint methylated band of $2.0 \mathrm{~kb}$ (arrows). Owing to an Mspl/Hpall restriction site polymorphism, which is irrelevant in this context, the unmethylated band is 0.9 or $0.5 \mathrm{~kb} .^{10}$ All the three AS patients are heterozygous for this polymorphism.

is semiquantitative and, as shown here, capable of detecting mosaic DNA methylation. Preliminary data suggest that methylation abnormalities can be detected even if the test sample contains up to 30\% DNA from normal cells (Nazlican, Zeschnigk, Lich, Gillessen-Kaesbach, Buiting \& Horsthemke, unpublished). However, exact degrees of methylation and limits of sensitivity can be determined by a quantitative study only (Nazlican et al., unpublished).

It has been suggested that the analysis of MS-PCR products by denaturing high-pressure liquid chromatography (DHPLC) is capable of detecting low cell mosaicism. ${ }^{7}$ Since DHPLC was used by one lab only, our data do not allow assessment of this method. It should be noted, however, that this lab obtained a correct result in both AS patients, and that a small peak corresponding to methylated DNA was detected in the 2002 AS patient.

Since $27 \%$ of patients with a sporadic-imprinting defect are mosaic for DNA methylation ${ }^{6}$ and this group contains patients with an atypical phenotype that overlaps with PWS, ${ }^{9}$ our results are highly relevant for diagnostic testing of patients suspected of having AS or PWS. Furthermore, a
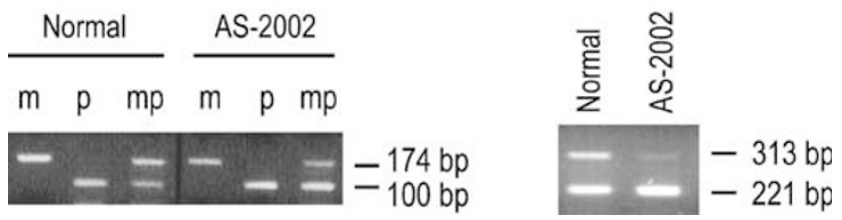

Figure 2 Typical MS-PCR results obtained with the Kubota test (a) and the Zeschnigk test (b). In both tests, the amplification products of the methylated allele are larger than the amplification products of the unmethylated allele. $\mathrm{m}$, simplex reaction with a primer pair specific for the methylated maternal allele; $p$, simplex reaction with a primer pair specific for the unmethylated paternal allele; $\mathrm{mp}$, duplex reaction with both primer pairs. Note that both the $m$ and $p$ reactions of the Kubota test are positive in the AS-2002 patient. In the Kubota duplex reactions, the methylated band of the patient is slightly fainter compared to the methylated band in the normal individual. In the Zeschnigk test, the methylated band is very faint, and the pattern is clearly different from that of the normal control.

mosaicism in AS and PWS can also be caused by other molecular mechanisms. It is worrying that more than $50 \%$ of the labs using the Kubota test obtained a false result. We strongly suggest the use of a duplex PCR (either according to Zeschnigk or to Kubota), and to carefully evaluate the relative intensities of the two bands.

\section{Acknowledgements}

We wish to thank Drs G Gillessen-Kaesbach, C Müller-Reible and the Berufsverband Medizinische Genetik for support.

Bernhard Horsthemke ${ }^{*, 1}$, Christina Lich ${ }^{1}$, Karin Buiting ${ }^{1}$, Roland Achmann ${ }^{2}$, Christa Aulehla-Scholz ${ }^{3}$, Alessandra Baumer $^{4}$, Joachim Bürger ${ }^{5}$, Bernd Dworniczak ${ }^{6}$, Dieter Gläser $^{7}$, Elke Holinski-Feder ${ }^{8}$, Bart Janssen ${ }^{9}$, Stephanie Kleinle $^{10}$, Lothar Kochhan ${ }^{11}$, Ernst Krasemann ${ }^{12}$, Cornelia Kraus $^{13}$, Peter Kroisel ${ }^{14}$, Hansjörg Plendl ${ }^{15}$, Sabine Purmann ${ }^{16}$, Gabriele Sander ${ }^{17}$, Heyko Skladny ${ }^{18}$, Eva Spitzer $^{19}$, Barbara Thamm-Mücke ${ }^{20}$, Raymonda VaronMateeva $^{21}$, Andreas Weinhäusel ${ }^{22}$, Helga Weirich ${ }^{23}$ 
${ }^{1}$ Institut für Humangenetik, Universitätsklinikum Essen, Germany;

${ }^{2}$ genteQ, Hamburg, Germany;

${ }^{3}$ Institut für Klinische Genetik, Olgahospital Stuttgart, Germany;

${ }^{4}$ Institut für Medizinische Genetik, Zürich, Switzerland;

${ }^{5}$ Münchener Rückversicherung, München, Germany;

${ }^{6}$ Institut für Humangenetik, Münster, Germany;

${ }^{7}$ Gregor Mendel Laboratorien, Neu-Ulm, Germany;

${ }^{8}$ Medizinisch-Genetisches Zentrum München, Germany;

${ }^{9}$ Institut für Humangenetik, Heidelberg, Germany;

${ }^{10}$ Labor Nevinny-Stickel, München, Germany;

${ }^{11}$ Labor Arndt und Partner, Hamburg, Germany;

${ }^{12}$ Labor Fenner, Hamburg, Germany;

${ }^{13}$ Institut für Humangenetik, Erlangen, Germany;

${ }^{14}$ Institut für Medizinische Biologie und Humangenetik, Graz,

Austria;

${ }^{15}$ Institut für Humangenetik, Kiel, Germany;

${ }^{16}$ Institut für Humangenetik, Lübeck, Germany;

${ }^{17}$ Landeskrankenanstalten Salzburg, Austria;

${ }^{18}$ Praxis Greiner, Mannheim, Germany;

${ }^{19}$ Berlin, Germany;

${ }^{20}$ Praxis Reising-Ackermann und Kollegen, Leipzig, Germany;

${ }^{21}$ Institut für Humangenetik, Charité Berlin, Germany;

${ }^{22}$ St Anna Kinderspital, Wien, Austria;

${ }^{23}$ Institut für Medizinische Biologie und Humangenetik, Innsbruck, Austria

*Correspondence: Dr Bernhard Horsthemke, Institut für Humangenetik, Universitätsklinikum Essen, Hufelandstrasse 55, D-45122 Essen, Germany. Tel: +49-201-7234556; Fax: + 49-201-7235900;

E-mail: b.horsthemke@uni-essen.de

\section{References}

1 Nicholls RD, Knepper JL: Genome organization, function, and imprinting in Prader-Willi and Angelman syndromes. Annu Rev Genomics Hum Genet 2001; 2: 153-175.

2 Zeschnigk M, Schmitz B, Dittrich B, Buiting K, Horsthemke B, Doerfler W: Imprinted segments in the human genome: DNA methylation patterns in the Prader-Willi/Angelman syndrome region as determined by the genomic sequencing method. Hum Mol Genet 1997; 6: 387-395.

3 Herman JG, Graff JR, Myohanen S, Nelkin BD, Baylin SB: Methylation-specific PCR: a novel PCR assay for methylation status of CpG islands. Proc Natl Acad Sci USA 1996; 93: 9821-9826.

4 Kubota T, Das S, Christian SL, Baylin SB, Herman JG, Ledbetter DH: Methylation-specific PCR simplifies imprinting analysis. Nat Genet 1997; 16: 16-17.

5 Zeschnigk M, Lich C, Buiting K, Doerfler W, Horsthemke B: A single-tube PCR test for the diagnosis of Angelman and PraderWilli syndrome based on allelic methylation differences at the SNRPN locus. Eur J Hum Genet 1997; 5: 94-98.

6 Buiting K, Gross S, Lich C, Gillessen-Kaesbach G, El-Maarri O, Horsthemke B: Epimutations in Prader-willi and Angelman syndromes: a molecular study of 136 patients with an imprinting defect. Am J Hum Genet 2003; 72: 571-577.

7 Baumer A, Wiedemann U, Hergersberg M, Schinzel A: A novel MSP/DHPLC method for the investigation of the methylation status of imprinted genes enables the molecular detection of low cell mosaicisms. Hum Mutat 2001; 17: 423-430.

8 Chotai KA, Payne SJ: A rapid, PCR based test for differential molecular diagnosis of Prader-Willi and Angelman syndromes. J Med Genet 1998; 35: 472-475.

9 Gillessen-Kaesbach G, Demuth S, Thiele H, Theile U, Lich C, Horsthemke B: A previously unrecognized phenotype characterized by obesity, muscular hypotonia, and ability to speak in patients with atypical Angelman syndrome caused by an imprinting defect. Eur J Hum Genet 1999; 7: 638-644.

10 Buiting K, Dittrich B, Groß S et al: Sporadic imprinting defects in Prader-Willi syndrome and Angelman syndrome: implications for imprint switch models, genetic counseling and prenatal diagnosis. Am J Hum Genet 1998; 63: 170-180. 